\title{
Using Hierarchical Models for 3D Human Body-Part Tracking
}

\author{
Leonid Raskin, Michael Rudzsky, and Ehud Rivlin \\ Computer Science Department, Technion, \\ Technion City, Haifa, Israel, 32000 \\ \{raskinl, rudzsky, ehudr\}@cs.technion.ac.il
}

\begin{abstract}
Human body pose estimation and tracking is a challenging task mainly because of the high dimensionality of the human body model. In this paper we introduce a Hierarchical Annealing Particle Filter (H-APF) algorithm for 3D articulated human body-part tracking. The method exploits Hierarchical Human Body Model (HHBM) in order to perform accurate body pose estimation. The method applies nonlinear dimensionality reduction combined with the dynamic motion model and the hierarchical body model. The dynamic motion model allows to make a better pose prediction, while the hierarchical model of the human body expresses conditional dependencies between the body parts and also allows us to capture properties of separate parts. The improved annealing approach is used for the propagation between different body models and sequential frames. The algorithm was checked on HumanEvaI and HumanEvaII datasets, as well as on other videos and proved to be effective and robust and was shown to be capable of performing an accurate and robust tracking. The comparison to other methods and the error calculations are provided.
\end{abstract}

\section{Introduction}

Human body pose estimation and tracking is a challenging task for several reasons. The large variety of poses and high dimensionality of the human 3D model complicates the examination of the entire subject and makes it harder to detect each body part separately. However, the poses can be presented in a low dimensional space using the dimensionality reduction techniques, such as Gaussian Process Latent Model (GPLVM) 1], locally linear embedding (LLE) [2], etc. The human motions can be described as curves in this space. This space can be obtained by learning different motion types [3]. However, such a reduction allows to detect poses similar to those, that were used for the learning process. In this paper we introduce a Hierarchical Annealing Particle Filter (H-APF) tracker, which exploits Hierarchical Human Body Model (HHBM) in order to perform accurate body part estimation. In this approach we apply a nonlinear dimensionality reduction using the Hierarchical Gaussian Process Latent Model (HGPLVM) [1] and the annealing particle filter [4. Hierarchical model of the human body expresses conditional dependencies between the body parts, but 
also allows us to capture properties of separate parts. Human body model state consists of two independent parts: one containing information about 3D location and orientation of the body and the other describing the articulation of the body. The articulation is presented as hierarchy of body parts. Each node in the hierarchy represent a set of body parts called partial pose. The method uses previously observed poses from different motion types to generate mapping functions from the low dimensional latent spaces to the data spaces, that correspond to the partial poses. The tracking algorithm consists of two stages. Firstly, the particles are generated in the latent space and are transformed to the data space using the learned mapping functions. Secondly, rotation and translation parameters are added to obtain valid poses. The likelihood function is calculated in order to evaluate how well these poses match the image. The resulting tracker estimates the locations in the latent spaces that represents poses with the highest likelihood. We show that our tracking algorithm is robust and provides good results even for the low frame rate videos. An additional advantage of the tracking algorithm is the ability to recover after temporal loss of the target.

\section{Related Works}

One of the commonly used technique for estimation the statistics of a random variable is the importance sampling. The estimation is based on samples of this random variable generated from a distribution, called the proposal distribution, which is easy to sample from. However, the approximation of this distribution for high dimensional spaces is a very computationally inefficient and hard task. Often a weighting function can be constructed according to the likelihood function, as it is in the CONDENSATION algorithm of Isard and Blake [5], which provides a good approximation of the proposal distribution and also is relatively easy to calculate. This method uses multiple predictions, obtained by drawing samples of pose and location prior and then propagating them using the dynamic model, which are refined by comparing them with the local image data, calculating the likelihood 5. The prior is typically quite diffused (because motion can be fast) but the likelihood function may be very peaky, containing multiple local maxima which are hard to account for in detail [6]. In such cases the algorithm usually detects several local maxima instead of choosing the global one. Annealed particle filter [4] or local searches are the ways to attack this difficulty. The main idea is to use a set of weighting functions instead of using a single one. While a single weighting function may contain several local maxima, the weighting functions in the set should be smoothed versions of it, and therefore contain a single maximum point, which can be detected using the regular annealed particle filter. The alternative method is to apply a strong model of dynamics [7]. The drawback of the annealed particle filter tracker is that the high dimensionality of the state space requires generation of a large amount of particles. In addition, the distribution variances, learned for the particle generation, are motion specific. This practically means that the tracker is applicable for the motion, that is used for the training. Finally, the APF is not robust and 
suffers from the lack of ability to detect a correct pose, once a target is lost (i.e. the body pose wrongly estimated).

In order to improve the trackers robustness, ability to recover from temporal target loss and in order to improve the computational effectiveness many researchers apply dimensionality reduction algorithm on the configuration space. There are several possible strategies for reducing the dimensionality. Firstly it is possible to restrict the range of movement of the subject [8]. But, due to the restricting assumptions, the resulting trackers are not capable of tracking general human poses. Another approach is to learn low-dimensional latent variable models [9]. However, methods like Isomap [10] and locally linear embedding (LLE) 2$]$ do not provide a mapping between the latent space and the data space, and, therefore Urtasun et al. [1] proposed to use a form of probabilistic dimensionality reduction by GPDM [12,13] to formulate the tracking as a nonlinear least-squares optimization problem. Andriluka et al. [14] use HGPLVM [1] to model prior on possible articulations and temporal coherency within a walking cycle. Raskin et al. [15] introduced Gaussian Process Annealed Particle Filter (GPAPF). According to this method, a set of poses is used in order to create a low dimensional latent space. This latent space is generated using Gaussian Process Dynamic Model (GPDM) for a nonlinear dimensionality reduction of the space of previously observed poses from different motion types, such as walking, running, punching and kicking. While for many actions it is intuitive that a motion can be represented in a low dimensional manifold, this is not the case for a set of different motions. Taking the walking motion as an example. One can notice that for this motion type the locations of the ankles are highly correlated with the location of the other body parts. Therefore, it seems natural to be able to represent the poses from this action in a low dimensional space. However, when several different actions are involved, the possibility of a dimensionality reduction, especially a usage of $2 \mathrm{D}$ and $3 \mathrm{D}$ spaces, is less intuitive.

This paper is organized as follows. Section 3 describes the tracking algorithm. Section 4 presents the experimental results for both tracking of different data sets and motion types. Finally, section 5 provides the conclusion and suggests the possible directions for the future research.

\section{Hierarchical Annealing Particle Filter}

The drawback of GPAPF algorithm is that a latent space is not capable of describing all possible poses. The space reduction must capture any dependencies between the poses of the different body parts. For example, if there is any connection between the parameters that describe the pose of the left hand and those, describing the right hand, then we can easily reduce the dimensionality of these parameters. However, if a person will perform a new movement, which differ from the learned ones, then the new poses will be represented less accurately by the latent space. Therefore, we suggest using a hierarchical model for the tracking. Instead of learning a single latent space that describes 
the whole body pose we use HGPLVM [1] to learn a hierarchy of the latent spaces. This approach allows us to exploit the dependencies between the poses of different body parts while accurately estimating of the pose of each part separately.

The commonly used human body model $\Gamma$ consists of 2 statistically independent parts $\Gamma=\{\Lambda, \Omega\}$. The first part $\Lambda \subseteq \mathbb{R}^{6}$ describes the body 3D location: the rotation and the translation. The second part $\Omega \subseteq \mathbb{R}^{25}$ describes the actual pose, which is represented by the angles between different body parts (see. 16 for more details about the human body model). Suppose the hierarchy consists of $H$ layers, where the highest layer (layer 1) represents the full body pose and the lowest layer (layer $H$ ) represents the separate body parts. Each hierarchy layer $h$ consists of $L_{h}$ latent spaces. Each node $l$ in hierarchy layer $h$ represents a partial body pose $\Omega_{h, l}$. Specifically, the root node describes the whole body pose; the nodes in the next hierarchy layer describe the pose of the legs, arms and the upper body (including the head); finally, the nodes in the last hierarchy layer describe each body part separately. Let us define $\Re\left(\Omega_{h, l}\right)$ as the set of the coordinates of $\Omega$ that are used in $\Omega_{h, l}$, where $\Omega_{h, l}$ is a subset of some $\Omega_{h-1, k}$ in the higher layer of the hierarchy. Such $k$ is denoted as $\tilde{l}$. For each $\Omega_{h, l}$ the algorithm constructs a latent spaces $\Theta_{h, l}$ and the mapping function $\wp^{(h, l)}: \Theta_{h, l} \mapsto \Omega_{h, l}$ that maps this latent space to the partial pose space $\Omega_{h, l}$. Let us also define $\theta_{h, l}$ as the latent coordinate in the $l$-th latent space in the $h$-th hierarchy layer and $\omega_{h, l}$ is the partial data vector that corresponds to $\theta_{h, l}$. Consequently, applying the definition of $\wp^{(h, l)}$ we have that $\omega_{h, l}=\wp^{(h, l)}\left(\theta_{h, l}\right)$. In addition for $\forall i$ we define $\Im(i)$ to be a pair $\langle h, l\rangle$, where $h$ is the lowest hierarchy layer and $l$ is the latent space in this layer, such that $i \in \Re\left(\Omega_{h, l}\right)$. In other words, $\Im(i)$ represent the lowest latent space in the hierarchy for which the $i$-th coordinate of $\Omega$ has been used in $\Omega_{h, l}$. Finally, $\lambda_{h, l, n}, \omega_{h, l, n}$ and $\theta_{h, l, n}$ are the location, pose vector and latent coordinates on the frame $n$ and hierarchy layer $h$ on the latent space $l$.

Now we present a Hierarchical Annealing Particle Filter (H-APF). A H-APF run is performed at each frame using image observations $y_{n}$. Following the notations used in [17] for the frame $n$ and hierarchy layer $h$ on the latent space $l$ the state of the tracker is represented by a set of weighted particles $S_{h, l, n}^{\pi}=\left\{\left(s_{h, l, n}^{(0)}, \pi_{h, l, n}^{(0)}\right), \ldots,\left(s_{h, l, n}^{(N)}, \pi_{h, l, n}^{(N)}\right)\right\}$. The un-weighted set of particles is denoted as $S_{h, l, n}=\left\{s_{h, l, n}^{(0)}, \ldots, s_{h, l, n}^{(N)}\right\}$. The state that is used contains translation, rotation values, latent coordinates and the full data space vectors: $s_{h, l, n}^{(i)}=\left\{\lambda_{h, l, n}^{(i)} ; \theta_{h, l, n}^{(i)} ; \omega_{h, l, n}^{(i)}\right\}$. The tracking algorithm consists of 2 stages. The first stage is the generation of new particles using the latent space. In the second stage the corresponding mapping function is applied that transforms latent coordinates to the data space. After the transformation, the translation and rotation parameters are added and the 31-dimensional vectors are constructed. These vectors represent a valid pose, which are projected to the cameras in order to estimate the likelihood. 
Each H-APF run has the following stages:

Step 1. For every frame hierarchical annealing algorithm run is started at layer $h=1$. Each latent space in each layer is initialized by a set of un-weighted particles $S_{h, l, n}$.

$$
S_{1,1, n}=\left\{\lambda_{1,1, n}^{(i)} ; \theta_{1,1, n}^{(i)} ; \omega_{1,1, n}^{(i)}\right\}_{i=1}^{N_{p}}
$$

Step 2. Calculate the weights of each particle:

$$
\begin{aligned}
& \pi_{h, l, n}^{(i)} \propto w^{m}\left(y_{n}, s_{h, l, n}\right)= \\
& k \frac{w^{m}\left(y_{n}, \lambda_{h, l, n}^{(i)}, \omega_{h, l, n}^{(i)}\right) p\left(\lambda_{h, l, n}^{(i)}, \theta_{h, l, n}^{(i)} \mid \lambda_{h, l, n}^{(i)}, \theta_{h, \tilde{l}, n}^{(i)}\right)}{q\left(\lambda_{h, l, n}^{(i)}, \theta_{h, l, n}^{(i)} \mid \lambda_{h, l, n}^{(i)}, \theta_{h, \tilde{l}, n}^{(i)}, y_{n}\right)}= \\
& k \frac{w^{m}\left(y_{n}, \Gamma_{h, l, n}^{(i)}\right) p\left(\lambda_{h, l, n}^{(i)}, \theta_{h, l, n}^{(i)} \mid \lambda_{h, l, n}^{(i)}, \theta_{h, \tilde{l}, n}^{(i)}\right)}{q\left(\lambda_{h, l, n}^{(i)}, \theta_{h, l, n}^{(i)} \mid \lambda_{h, l, n}^{(i)}, \theta_{h, \tilde{l}, n}^{(i)}, y_{n}\right)}
\end{aligned}
$$

where $w^{m}\left(y_{n}, \Gamma\right)$ is the weighting function suggested by Deutscher and Reid [17] and $k$ is a normalization factor so that $\sum_{i=1}^{N_{p}} \pi_{n}^{(i)}=1$. The weighted set, that is constructed, will be used to draw particles for the next layer.

Step 3. $N$ particles are drawn randomly with replacements and with a probability equal to their weight $\pi_{h, l, n}^{(i)}$. For every latent space $l$ in the hierarchy level $h+1$ the particle $s_{h+1, l, n}^{(j)}$ is produces using the $j^{t h}$ chosen particle $s_{h, \hat{l}, n}^{(j)}(\hat{l}$ is the index of the parent node in the hierarchy tree):

$$
\begin{gathered}
\lambda_{h+1, l, n}^{(j)}=\lambda_{h, \hat{l}, n}^{(j)}+B_{\lambda_{h+1}} \\
\theta_{h+1, l, n}^{(j)}=\phi\left(\theta_{h, \hat{l}, n}^{(j)}\right)+B_{\theta_{h, \hat{l}}}
\end{gathered}
$$

In order to construct a full pose vector $\omega_{h+1, l, n}^{(j)}$ is initialized with the $\omega_{h, \hat{l}, n}^{(j)}$

$$
\omega_{h+1, l, n}^{(j)}=\omega_{h, \hat{l}, n}^{(j)}
$$

and then updated on the coordinates defined by $\Omega_{h+1, l}$ using the new $\theta_{h+1, l, n}^{(j)}$

$$
\left.\left(\omega_{h+1, l, n}^{(j)}\right)\right|_{\Omega_{h+1, l}}=\wp^{h+1, l}\left(\theta_{h+1, l, n}^{(j)}\right)
$$

(The notation $\left.a\right|_{B}$ stands for the coordinates of vector $a \in A$ defined by the subspace $B \subseteq A$.) The idea is to use a pose that was estimated using the higher 
hierarchy layer, with small variations in the coordinates described by the $\Omega_{h+1, l}$ subspace.

Finally, the new particle for the latent space $l$ in the hierarchy level $h+1$ is:

$$
s_{h+1, l, n}^{(j)}=\left\{\lambda_{h+1, l, n}^{(j)} ; \omega_{h+1, l, n}^{(j)} ; \theta_{h+1, l, n}^{(j)}\right\}
$$

The $B_{\lambda_{h}}$ and $B_{\theta_{h, l}}$ are multivariate Gaussian random variables with covariances and $\Sigma_{\lambda_{h}}$ and $\Sigma_{\theta_{h, l}}$ correspondingly and mean 0 .

Step 4. The sets $S_{h+1, l, n}$ have now been produced which can be used to initialize the layer $h+1$. The process is repeated until we arrive to the $H$-th layer.

Step 5. The $j^{\text {th }}$ chosen particle $s_{H, l, n}^{(j)}$ in every latent space $l$ in the lowest hierarchy level and their ancestors (the particles in the higher layers that used to produce $s_{H, l, n}^{(j)}$ are used to produce $s_{1,1, n+1}^{(j)}$ un-weighted particle set for the next observation:

$$
\begin{aligned}
& \lambda_{1,1, n+1}^{(j)}=\frac{1}{L_{H}} \sum_{l=1}^{L_{H}} \lambda_{H, l, n}^{(j)} \\
& \forall i \omega^{(j)}(i)=\widehat{\omega}_{\Im(i), n}^{(j)} \\
& \theta_{1,1, n+1}=\wp_{1,1}^{-1}\left(\omega_{1,1, n+1}^{(j)}\right)
\end{aligned}
$$

Here $\widehat{\omega}_{h, k, n}^{(j)}$ denotes an ancestor of $\omega_{H, l, n}^{(j)}$ in $h$-th layer of the hierarchy.

Step 6. The optimal configuration can be calculated using the following method:

$$
\begin{aligned}
& \lambda_{n}^{(o p t)}=\frac{1}{L_{H}} \sum_{l l=1}^{L_{H}} \sum_{j=1}^{N} \lambda_{H, l, n}^{(j)} \pi_{h, l, n}^{(j)} \\
& \forall i \omega^{(j)}(i)=\widehat{\omega}_{\Im(i), n}^{(j)} \\
& \omega_{n}^{(o p t)}=\sum_{j=1}^{N} \omega^{(j)} \pi^{(j)}
\end{aligned}
$$

where, similar to stage $2, \pi^{(j)}=w^{m}\left(y_{n},\left\langle\lambda_{n}^{(o p t)}, \omega^{(j)}\right\rangle\right)$ is the normalized weighting function so that $\sum_{i=1}^{N_{p}} \pi^{(i)}=1$.

\section{Results}

We have tested H-APF tracker using the HumanEvaI and HumanEvaII datasets [18. The sequences contain different activities, such as walking, boxing, jogging etc., which were captured by several synchronized and mutually calibrated cameras. The sequences were captured using the MoCap system that provides the correct 3D locations of the body joints, such as shoulders and knees. This information is used for evaluation of the results and comparison to other tracking 


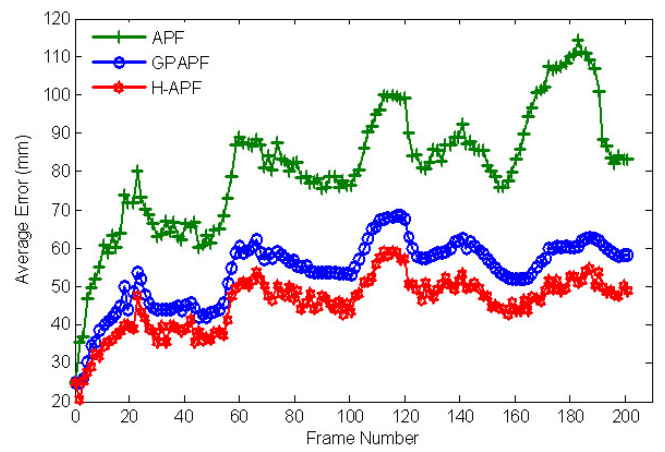

Fig. 1. The errors of the APF tracker (green crosses), GPAPF tracker (blue circles) and H-APF tracker (red stars) for a walking sequence captured at $15 \mathrm{fps}$

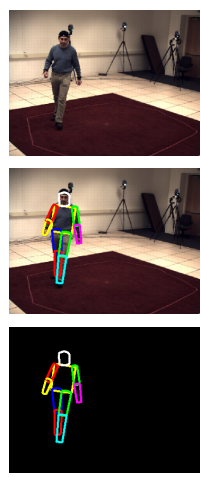

frame 50
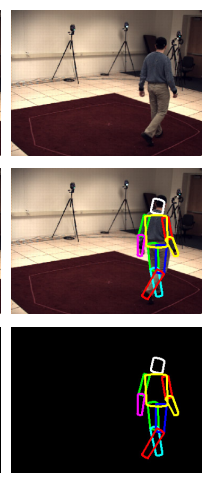

frame 230

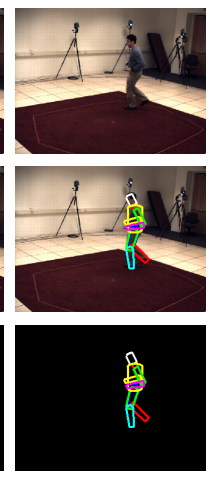

frame 640

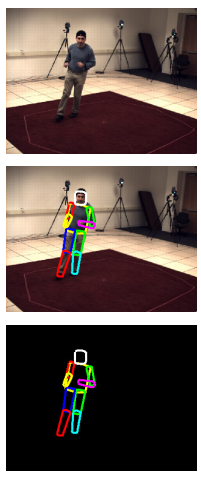

frame 700

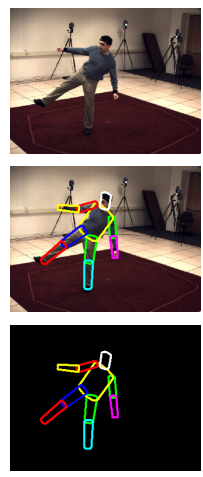

frame 800

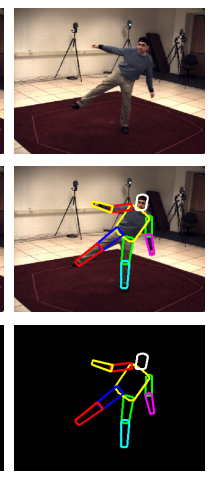

frame 1000

Fig. 2. Tracking results of H-APF tracker. Sample frames from the combo1 sequence from HumanEvaII(S2) dataset.

algorithms. The error is calculated, based on comparison of the tracker's output to the ground truth, using average distance in millimeters between 3-D joint locations [16].

The first sequence that we have used contain a person, walking in a circle. The video was captured at $60 \mathrm{fps}$ frame rate. We have compared the results produced by APF, GPAPF and H-APF trackers. For each algorithm we have used 5 layers, with 100 particles in each. Fig. 1 shows the error graphs, produced by APF (green crosses), the GPAPF (blue circles) and the H-APF (red stars) trackers. We have also tried to compare our results to the results of CONDENSATION algorithm. However, the results of that algorithm were either very poor or very large number of particles needed to be used, which made this algorithm computationally not effective. Therefore we do not provide the results of this comparison. 

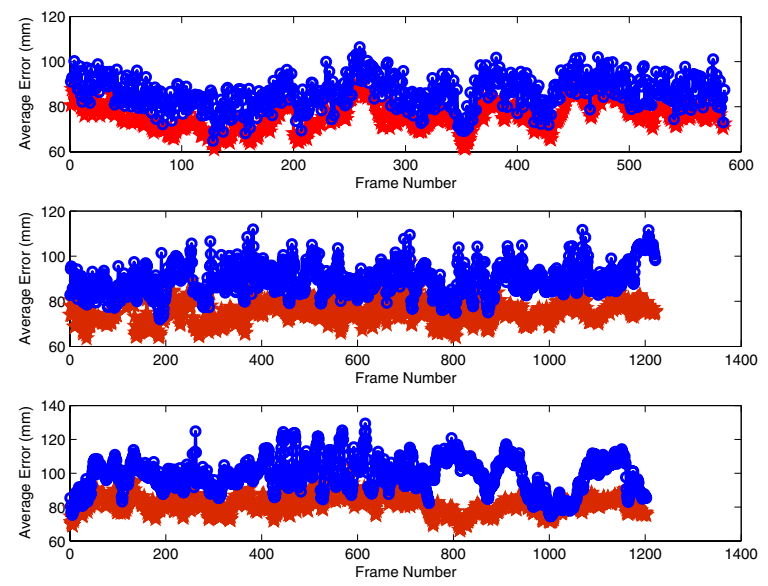

Fig. 3. The errors for HumanEvaI(S1, walking1, frames 6-590)(top), HumanEvaII(S2, frames 1-1202)(middle) and HumanEvaII(S4, frames 2-1258)(bottom). The errors produced by GPAPF tracker are marked by blue circles and the error of the H-APF tracker are marked by red stars.
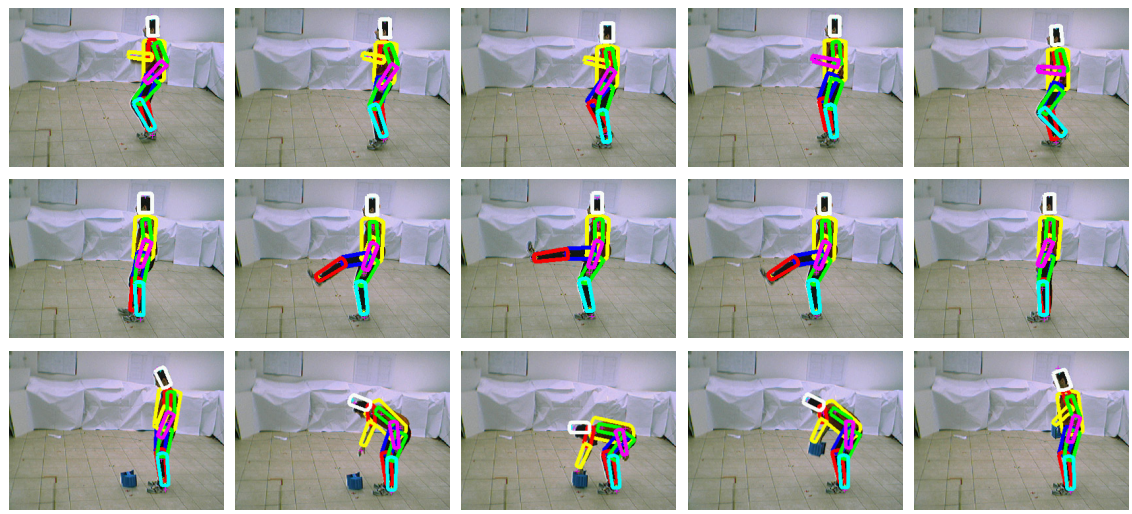

Fig. 4. Tracking results of H-APF tracker. Sample frames from the running, kicking and lifting an object sequences.

Next we trained HGPLVM with several different motion types. We used this latent space in order to track the body parts on the videos from the $\mathrm{Hu}-$ manEvaI and HumanEvaII datasets. Fig. 2 shows the result of the tracking of the HumanEvaII(S2) dataset, which combines 3 different behaviors: walking, jogging and balancing and Fig. 3 presents the errors for HumanEvaI(S1, walking1, frames 6-590)(top), HumanEvaII(S2, frames 1-1202)(middle) and $\mathrm{Hu}-$ manEvaII(S4, frames 2-1258)(bottom). Finally, Fig. 4 shows the results from the running, kicking and lifting an object sequences. 


\section{Conclusion and Future Work}

In this paper we have introduced an approach that uses HGPLVM to improve the ability of the annealed particle filter tracker to track the object even in a high dimensional space. The usage of hierarchy allows better detect body part position and thus perform more accurate tracking.

An interesting problem is to perform tracking of the interactions between multiple actors. The main problem is constructing a latent space. While a single persons poses can be described using a low dimensional space it may not be the case for multiple people. The other problem here is that in this case there is high possibility of occlusion. Furthermore, while for a single person each body part can be seen from at least one camera that is not the case for the crowded scenes.

\section{References}

1. Lawrence, N.D., Moore, A.J.: Hierarchical gaussian process latent variable models. In: Proc. International Conference on Machine Learning (ICML) (2007)

2. Roweis, S.T., Saul, L.K.: Nonlinear dimensionality reduction by locally linear embedding. Science 290, 2323-2326 (2000)

3. Elgammal, A.M., Lee, C.: Inferring 3D body pose from silhouettes using activity mani-fold learning. In: Proc. Computer Vision and Pattern Recognition (CVPR), vol. 2, pp. 681-688 (2004)

4. Deutscher, J., Blake, A., Reid, I.: Articulated body motion capture by annealed particle filtering. In: Proc. Computer Vision and Pattern Recognition (CVPR), pp. 2126-2133 (2000)

5. Isard, M., Blake, A.: Condensation - conditional density propagation for visual tracking. International Journal of Computer Vision (IJCV) 29(1), 5-28 (1998)

6. Sidenbladh, H., Black, M.J., Fleet, D.: Stochastic tracking of 3D human figures using 2D image motion. In: Vernon, D. (ed.) ECCV 2000. LNCS, vol. 1843, pp. 702-718. Springer, Heidelberg (2000)

7. Mikolajczyk, K., Schmid, K., Zisserman, A.: Human detection based on a probabilistic assembly of robust part detectors. In: Pajdla, T., Matas, J. (eds.) ECCV 2004. LNCS, vol. 3021, pp. 69-82. Springer, Heidelberg (2004)

8. Rohr, K.: Human movement analysis based on explicit motion models. MotionBased Recognition 8, 171-198 (1997)

9. Wang, Q., Xu, G., Ai, H.: Learning object intrinsic structure for robust visual tracking. In: Proc. Computer Vision and Pattern Recognition (CVPR), vol. 2, pp. 227-233 (2003)

10. Tenenbaum, J.B., de Silva, V., Langford, J.C.: A global geometric framework for nonlinear dimensionality reduction. Science 290, 2319-2323 (2000)

11. Urtasun, R., Fleet, D.J., Fua, P.: 3D people tracking with gaussian process dynamical models. In: Proc. Computer Vision and Pattern Recognition (CVPR), vol. 1, pp. 238-245 (2006)

12. Lawrence, N.D.: Gaussian process latent variable models for visualization of high dimensional data. In: Advances in Neural Information Processing Systems (NIPS), vol. 16, pp. 329-336 (2004)

13. Wang, J., Fleet, D.J., Hetzmann, A.: Gaussian process dynamical models. In: Information Processing Systems (NIPS), pp. 1441-1448 (2005) 
14. Andriluka, M., Roth, S., Schiele, B.: People-tracking-by-detection and peopledetection-by-tracking. In: Proc. Computer Vision and Pattern Recognition (CVPR), vol. 1, pp. 1-8 (2008)

15. Raskin, L., Rudzsky, M., Rivlin, E.: Dimensionality reduction for articulated body tracking. In: Proc. The True Vision Capture, Transmission and Display of 3D Video (3DTV) (2007)

16. Balan, A., Sigal, L., Black, M.: A quantitative evaluation of video-based 3D person tracking. In: IEEE Workshop on Visual Surveillance and Performance Evaluation of Tracking and Surveillance (VS-PETS), pp. 349-356 (2005)

17. Deutscher, J., Reid, I.: Articulated body motion capture by stochastic search. International Journal of Computer Vision (IJCV) 61(2), 185-205 (2004)

18. Sigal, L., Black, M.J.: Measure locally, reason globally: Occlusion-sensitive articulated pose estimation. In: Proc. Computer Vision and Pattern Recognition (CVPR), vol. 2, pp. 2041-2048 (2006) 